\title{
HIDROGENAÇÃO DO BIODIESEL COM CATALISADORES A BASE DE COBRE
}

\author{
I. B. T. de MACEDO ${ }^{1}$, A. C. STAFUSSA ${ }^{1}$, S. H. B. De FARIA ${ }^{1}$, O. A. A. Dos SANTOS ${ }^{1}$, \\ L. M. M. JORGE ${ }^{1}$ \\ ${ }^{1}$ Universidade Estadual de Maringá, Departamento de Engenharia Química \\ E-mail para contato: lmmjorge@uem.br
}

\begin{abstract}
RESUMO - Uma alternativa a combustíveis derivados de petróleo é o biodiesel, este apresenta como vantagens menor emissão de poluentes e biodegrabilidade. Porém, um dos principais problemas técnicos enfrentados pelo biodiesel é a sua suscetibilidade à oxidação. $\mathrm{O}$ processo de hidrogenação catalítica pode ser aplicado com a finalidade de melhorar a estabilidade oxidativa do biodiesel, produzindo um combustível de propriedades otimizadas. Com o propósito de melhorar a estabilidade frente à oxidação, este trabalho tem como finalidade apresentar o estudo da reação de hidrogenação catalítica do biodiesel. Para isso foram realizadas reações em diferentes condições de pressão e quantidade de catalisador, utilizando para tal, um catalisador à base de cobre. A fim de caracterizar os produtos, estes foram analisados quanto à estabilidade oxidativa à $110^{\circ} \mathrm{C}$, índice de iodo e viscosidade. Os resultados indicam que a hidrogenação do biodiesel nas condições experimentais exploradas não favoreceu uma melhora significativa na sua estabilidade oxidativa.
\end{abstract}

\section{INTRODUÇÃO}

A utilização de óleos vegetais como combustíveis foi sugerida pela primeira vez em 1900, por Rudolf Diesel (1853-1913), inventor do motor a diesel. Contudo, em razão dos baixos custos e da disponibilidade de petróleo, o diesel mineral foi adotado como o principal combustível para utilização nos motores (Pousa et al., 2007). O uso de óleos vegetais como alternativa de fatores limitantes, como alta viscosidade, combustão incompleta e baixa volatilidade que resulta na formação de depósitos nos injetores de combustível das máquinas. Para superá-los, os triacilgliceróis devem ser manipulados de maneira a tornarem-se compatíveis com as máquinas existentes (Geris et al., 2007). O biodiesel é vantajoso pelo fato de poder ser produzido a partir de uma grande variedade de matérias-primas, e dependendo da matéria prima utilizada pode apresentar qualidades diferentes.

O processo oxidativo sofrido pelo biodiesel, dificulta a armazenagem do combustível. Alguns fatores que contribuem diretamente para essa degradação são: luz, temperatura, ação do ar, umidade e presença de metais catalíticos (Russo, 2013). Um dos motivos da sua instabilidade oxidativa é o alto teor de cadeias alquílicas poli-insaturadas. Essas poli-insaturações aceleram a autoxidação devido à presença de posições alílicas e dialílicas em suas cadeias carbônicas, que estabilizam para equipamentos a diesel é considerado insatisfatório e impraticável, por apresentar uma série o intermediário radicalar. (Carvalho, 2008). 
Visando aplicações tecnológicas, é de suma importância que o biodiesel seja estabilizado antes da sua comercialização. Vários podem ser os métodos para estabilização, sendo a hidrogenação um deles. No processo de hidrogenação, parte das duplas ligações é saturada, enquanto algumas duplas são isomerizadas de cis para trans. Portanto, o biodiesel é passível de ser hidrogenado para aumentar sua longevidade tanto no transporte, armazenamento e combustão sem perdas de qualidade. (Zhang et al., 2003).

A hidrogenação do biodiesel altera suas propriedades físicas e químicas, modificando sua viscosidade, tensão superficial e seu ponto de fusão, principalmente. O biodiesel hidrogenado apresenta um índice de cetano mais elevado, aproximando-se do diesel mineral (Carvalho, 2008). Um número adequado de cetano no combustível traz vantagens como o favorecimento do bom funcionamento do motor. É necessário o controle do processo de hidrogenação para obter as propriedades físicas e químicas desejadas no biodiesel.

O índice de iodo, um importante parâmetro de qualidade, determina a quantidade de ácidos graxos insaturados presentes no óleo vegetal e está relacionado diretamente com a estabilidade do biodiesel. Basicamente o número representa a massa de iodo (em gramas) necessária para saturar os ácidos graxos não saturados. Como consequências da oxidação, pode ocorrer a formação de depósitos e a deterioração do óleo lubrificante, influenciando no desempenho do motor pela elevação da sua viscosidade e da acidez (Ferrari et al., 2005).

Na hidrogenação, os catalisadores homogêneos mais utilizados são complexos dos metais de transição solúveis no óleo, enquanto na catálise heterogênea utilizam-se metais como Pd, Pt, $\mathrm{Cu}, \mathrm{Ag}, \mathrm{Rh}$, Ir, Os e Ru suportados em sílica (Fernandez et al., 2007), alumina (Piqueras et al., 2006) e carbono (Hsu et al,. 1986). Estudos como o de He et al (2013) apontam para a possibilidade da utilização com sucesso de catalisadores a base de $\mathrm{Cu}$ no processo de hidrogenação de diferentes moléculas orgânicas de origem vegetal.

O presente trabalho tem como objetivo, o estudo da possibilidade do uso de catalisadores a base de cobre no processo de hidrogenação seletiva do biodiesel, além da análise e caracterização do biodiesel resultante e contribuir para a definição de parâmetros ótimos da reação de hidrogenação com catalisadores heterogêneos a base de cobre.

\section{METODOLOGIA}

O equipamento utilizado para o processo de hidrogenação é constituído basicamente de duas partes, um sistema de alimentação de gás reagente (BRGDS fabricado pela Autoclave Engineer) e por um reator do tipo autoclave (PARR). O sistema permite fornecer gás reagente e gás inerte para o reator de aço inoxidável, possui válvulas para extração de amostras e regulagem da pressão interna do reator, também contem um eficiente sistema de agitação e um controlador de temperatura programável ligado ao aquecedor. $\mathrm{O}$ biodiesel utilizado neste trabalho foi fornecido pela empresa BsBios. O metanol foi escolhido como solvente devido a sua alta solubilidade em $\mathrm{H}_{2}$ conforme indicado nos estudos de Linke e Seidell (1958).

Para realizar as reações propostas, o catalisador comercial R-120 a base de cobre, que se apresentava na forma de pellets foi tranformado à pó, então foi ativado num reator de leito fixo, a uma temperatura de $300{ }^{\circ} \mathrm{C}$ sob fluxo de uma mistura gasosa (5\% de hidrogênio e $95 \%$ de nitrogênio), durante 3 horas. Posteriormente, o catalisador foi imediatamente vertido em um 
recipiente contendo heptano, minimizando o contato deste com o ar e mantido a baixas temperaturas. No preparo do catalisador para os ensaios, houve a secagem sob um fluxo de nitrogênio gasoso até que este se apresentasse como um pó seco. Após pesado, pode-se adicionar este ao biodiesel para posterior adição no reator.

Portanto, o solvente, catalisador e o biodiesel foram devidamente pesados e colocados dentro do vaso reacional. Foram realizadas reações para diferentes condições de pressão e quantidades de catalisador. Mantendo-se constante o tempo de reação (3h) e a temperatura (200 ${ }^{\circ} \mathrm{C}$ ). Foram realizados 10 ensaios experimentais, mediante a adição de $50 \mathrm{~g}$ de biodiesel e $50 \mathrm{~g}$ de metanol, na proporção 1:1. As concentrações de catalisador em massa de biodiesel e as pressões as quais as amostras foram submetidas estão apresentadas na Tabela 1.

Tabela 1 - Condições discriminadas para as reações 1:1.

\begin{tabular}{|c|c|c|c|c|c|}
\hline Reação & $\begin{array}{c}\text { Pressão } \\
\quad(P s i)\end{array}$ & $\begin{array}{c}\text { Quantidade de } \\
\text { catalisador em massa } \\
\text { de biodiesel (\%) }\end{array}$ & Reação & $\begin{array}{c}\text { Pressão } \\
(\text { Psi })\end{array}$ & $\begin{array}{c}\text { Quantidade de } \\
\text { catalisador em massa } \\
\text { de biodiesel (\%) }\end{array}$ \\
\hline 1 & \multirow{5}{*}{500} & 0 & 6 & \multirow{5}{*}{750} & 0 \\
\hline 2 & & 1 & 7 & & 1 \\
\hline 3 & & 2 & 8 & & 2 \\
\hline 4 & & 3 & 9 & & 3 \\
\hline 5 & & 4 & 10 & & 4 \\
\hline
\end{tabular}

Após a adição do catalisador e dos reagentes, o reator foi hermeticamente fechado e em seguida fez-se a purga com gás hidrogênio. O aquecimento do reator foi feito sob agitação até que este alcançasse a temperatura desejada. Em seguida, o hidrogênio foi injetado no reator via válvulas de controle, sendo que a pressão fica constante durante todo o tempo da reação. Ao fim das 3 horas de reação e a mistura resfriada, o reator é despressurizado. Retira-se os produtos da reação e armazena-se em baixa temperatura, até as análises pertinentes. Posteriormente, todos os produtos da reação foram devidamente preparados para as análises e lavados com água e ácido clorídrico, com a finalidade de remover o catalisador e metanol e obter amostras límpidas e livres de impurezas. Quando necessário, as amostras também eram submetidas a centrifugação para retirada do catalisador residual.

Foram efetuados três tipos de análises: índice de iodo, estabilidade oxidativa e viscosidade do combustível. A viscosidade dinâmica de cada amostra foi obtida a partir do viscosímetro de bolas (Brookfield-modelo KF40), a estabilidade oxidativa foi determinada a partir de análises em um laboratório particular, com equipamentos específicos e segundo a especificação EN14112 e para determinação do índice de iodo, utilizou-se a metodologia segundo Wijs. Os resultados foram comparados àqueles do biodiesel não-hidrogenado.

\section{RESULTADOS E DISCUSSÕES}




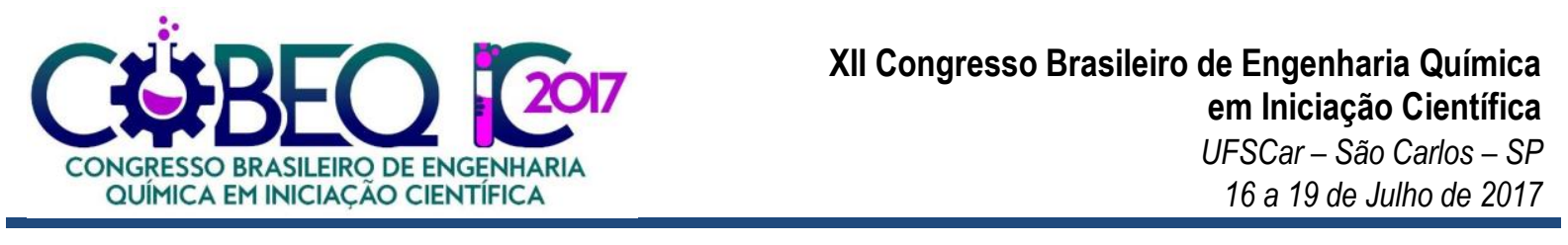

A Tabela 2 contém os resultados obtidos a partir das análises das reações e o da amostra padrão (biodiesel não-hidrogenado).

Tabela 2 - Resultados encontrados para as amostras reacionais.

\begin{tabular}{|c|c|c|c|}
\hline Amostras & $\begin{array}{c}\text { Viscosidade cinemática à } \\
\mathbf{4 0}\left({ }^{\circ} \mathbf{C}\right) \mathbf{m m}^{2} / \mathbf{s}\end{array}$ & $\begin{array}{c}\text { Estabilidade Oxidativa } \\
(\boldsymbol{h})\end{array}$ & Índice de iodo (\% massa) \\
\hline Padrão & 3,54 & 8,7 & 68,64 \\
\hline $\mathbf{1}$ & 3,27 & 0,04 & 143,43 \\
\hline $\mathbf{2}$ & 3,32 & 0,06 & 153,35 \\
\hline $\mathbf{3}$ & 3,21 & 0,11 & 135,47 \\
\hline $\mathbf{4}$ & 3,18 & 0,05 & 155,96 \\
\hline $\mathbf{5}$ & 3,27 & 0,06 & 165,10 \\
\hline $\mathbf{6}$ & 3,23 & 0,05 & 150,15 \\
\hline $\mathbf{7}$ & 3,28 & 0,07 & 162,00 \\
\hline $\mathbf{8}$ & 3,26 & 0,07 & 151,76 \\
\hline $\mathbf{9}$ & 3,23 & 0,09 & 163,92 \\
\hline $\mathbf{1 0}$ & 3,24 & 0,04 & 151,72 \\
\hline
\end{tabular}

Os resultados de viscosidades cinemáticas se apresentam dentro da faixa considerada ideal para o biodiesel, sendo que a amostra 4 teve o menor valor analisado, o qual é de 3,18 $\mathrm{mm}^{2} / \mathrm{s}$ e a 2 obteve o maior valor de viscosidade: $3,32 \mathrm{~mm}^{2} / \mathrm{s}$. O restante das amostras se mostraram dentro desse intervalo. Não houve uma correlação linear nos dados, porém neste ponto a reação se mostrou benéfica, já que o valor da viscosidade decaiu do valor inicial.

Quanto ao índice de iodo, de acordo com a legislação brasileira, resolução ANP $n^{\circ} 42$, não existe um limite máximo estabelecido. Porém deve ser registrado seu valor. Na prática, sabe-se que um índice de iodo acima de 135 ( $\mathrm{g} \mathrm{I}_{2} / 100 \mathrm{~g}$ ) para combustíveis não é satisfatório. Os valores encontrados nas análises foram muito maiores em relação a amostra padrão, sendo que quanto maior o grau de insaturações do óleo, maior o índice de iodo e isto vem de encontro ao fato da estabilidade oxidativa ter se mostrado baixa.

Apesar dos dados do tempo de estabilidade terem se mostrado muito superiores em comparação ao da amostra que não foi submetida à hidrogenação (padrão), há uma relação entre a pressão utilizada nos ensaios e as outras variáveis. Nas concentrações mais baixas de catalisador a pressão de 750 Psi se mostra mais favorável, enquanto na concentração de $2 \%$ há uma inversão e a reação é mais favorecida à 500 Psi. É provável que exista um ponto ótimo de operação e controle da reação, próximo a concentração intermediária de catalisador, que se 
apresente mais vantajoso à estabilidade do biodiesel. Sendo que na maior concentração utilizada, os valores não foram satisfatórios a ponto de se tornarem viáveis.

Aparentemente tanto a pressão como a concentração de catalisador exercem pouca ou nenhuma influência sobre o índice de iodo (Figura 1) e a estabilidade oxidativa a $110^{\circ} \mathrm{C}$ (Figura 2), conforme pode-se constatar analisando-se o comportamento os dados experimentais e das linhas de tendência (traços pontilhados).

Figura 1 - Índice de iodo em função da concentração de catalisador

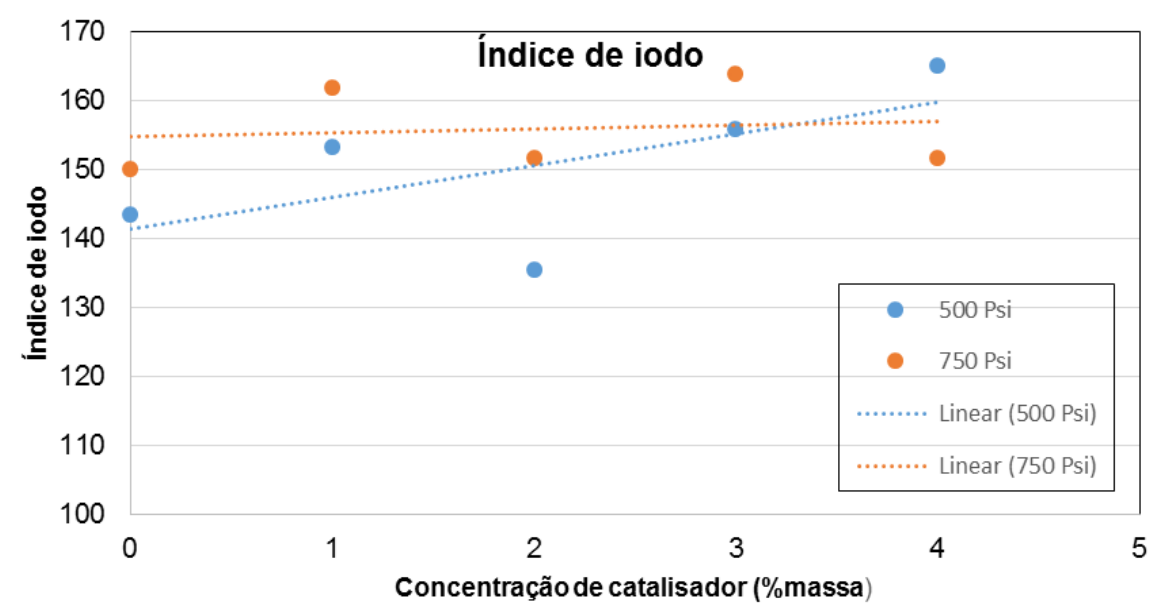

Figura 2 - Influência da pressão e da concentração de catalisador sobre a estabilidade

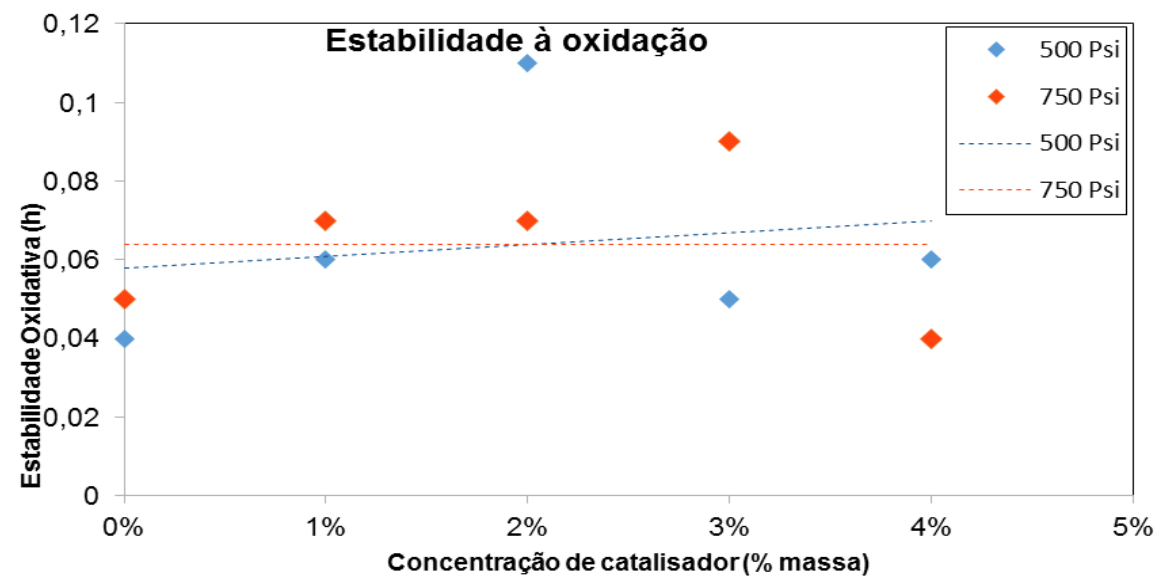

Alguns fatores podem ter contribuído para que os valores encontrados fossem muito abaixo do esperado. Há hipóteses da ocorrência de uma hidrogenação total, a qual leva a uma mistura de ésteres alquílicos de ácidos graxos com reduzida fluidez a baixas temperaturas e alto ponto de cristalização, comprometendo o seu uso. Sabe-se também que contaminantes metálicos podem reduzir a estabilidade oxidativa de óleos e gorduras. Os processos de lavagem das amostras, as condições de estocagem e/ou utilização de solvente também podem ter promovido efeitos negativos. Entretanto, em comparação com as reações em branco, as reações com catalisador, em sua maioria, foram satisfatórias à melhoria da estabilidade à oxidação do biodiesel. As amostras sem adição de catalisador se mostraram com menor tempo de estabilidade oxidativa comparadas ao biodiesel padrão, levando ao descarte da suposição de 
contaminantes metálicos. Tal fato indica a possibilidade do uso de catalisadores a base de cobre na hidrogenação, desde que se tenha um aperfeiçoamento das condições experimentais.

\section{CONCLUSÃO}

Os ensaios de hidrogenação catalítica do biodiesel foram realizados em diferentes condições experimentais a fim de se obter os melhores parâmetros para realizar as reações com catalisadores a base de cobre, porém os resultados obtidos mostraram que a reação de hidrogenação não promoveu melhora significativa sobre as propriedades do biodiesel comercial não hidrogenado (padrão). Tanto a pressão como a concentração de catalisador pouco afetaram a estabilidade oxidativa do biodiesel hidrogenado. Os resultados obtidos sugerem a necessidade do aprofundamento dos estudos nesta área a fim de precisar as condições ótimas de hidrogenação e eliminar os possíveis fatores prejudiciais a este processo.

\section{REFERÊNCIAS}

CARVALHO, M. de S. Hidrogenação seletiva do biodiesel de soja em sistema bifásico utilizando líquido iônico BMI.BF4. 2008. 71f. Dissertação (Mestrado em Química)- Universidade de Brasília, Instituto de Química, Brasília, 2008.

FERRARI, R.A.; OLIVEIRA, V.S.; SCABIO, A. Oxidative stability of biodiesel from soybean oil fatty acid ethyl esters. Sci. agric., v.62, n.3, pp. 291-295, 2005.

FERNANDEZ, M.B.; et al. Journal of Food Engineering, 2007, 82, 199.

GERIS, R., SANTOS, N. A., AMARAL, B. A., MAIA, I. D., DOURADO, V., CARVALHO, J. R. Biodiesel de Soja - Reação de Transesterificação Para Aulas Práticas de Química. Química Nova, v. 30, p. 1369-1373, 2007.

HE, L., CHENG, H., LIANG, G., YU, Y., ZHAO, F., Effect of structure of CuO/ZnO/Al2O3 composites on catalytic performance for hydrogenation of fatty acid ester. Applied Catalysis A: General, v. 452, p. 88-93, 2013.

HSU, N.; DIOSADY L. L.; GRAYDON W. F.; RUBIN L. J. Heterogeneous catalytic hydrogenation of canola oil using palladium. J. Am. Oil Chem. Soc. 1986, 63, 1036.

LINKE, W. F.; SEIDELL, A. Solubilities of Inorganic and Metal-Organic Compounds, vol. 1. American Chemical Society, 2008. p. 1075

PIQUERAS, C.; BOTTINI, S.; DAMIANI, D. Applied Catalysis A: General, 2006, 313, 177.

POUSA, G. P. A., SANTOS, A. L. F., SUAREZ P. A. Z., History and policy of biodiesel in Brazil. Energy Policy, v. 35, p. 5393-5398, 2007.

RUSSO, M. E. Estudo do comportamento oxidativo do biodiesel de soja. 2013. 78f. Dissertação (mestrado) - Universidade Estadual do Centro-Oeste, Guarapuava, 2013.

ZHANG, Y.; DUBÉ, M.A.; MCLEAN, D.D.; KATES, M. Biodiesel production from waste cooking oil. Bioresour. Technol. 2003, 89, 1. 